\title{
Epidemiologia dos acidentes ofídicos notificados pelo Centro de Assistência e Informação Toxicológica de Campina Grande (Ceatox-CG), Paraíba
}

\section{Epidemiologic profile of snakebites reported by the Poison Information Center of Campina Grande, Paraíba}

\author{
Josiverton de Carvalho Lemos ${ }^{1}$ \\ Thaise Dantas de Almeida ${ }^{2}$ \\ Sayonara Maria Lia Fook ${ }^{3}$ \\ Adriana de Azevedo Paiva ${ }^{4}$ \\ Mônica Oliveira da Silva Simões ${ }^{5}$ \\ ${ }^{1}$ Centro de Atendimento Toxicológico de Campina Grande (Ceatox-CG). \\ Departamento de Farmácia da Universidade Estadual da Paraíba, Campina \\ Grande, PB \\ ${ }^{2}$ Departamento de Saúde Coletiva da Universidade Estadual da Paraíba, \\ Campina Grande, PB \\ ${ }^{3}$ Departamento de Farmácia da Universidade Estadual da Paraíba, Campina \\ Grande, PB. Centro de Atendimento Toxicológico de Campina Grande, PB \\ ${ }^{4}$ Núcleo de Estudos e Pesquisas Epidemiológicas - NEPE. Universidade Estadual \\ da Paraíba, Campina Grande, PB \\ ${ }^{5}$ Departamento de Farmácia da Universidade Estadual da Paraíba, Campina \\ Grande, PB
}

O projeto que deu origem ao artigo foi avaliado e aprovado pelo Comitê de Ética da Universidade Estadual da Paraíba e registrado no Sistema Nacional de Informação sobre Ética em Pesquisa (Sisnep) com o CAAE-0006.0.133.000-06.

Correspondência: Josiverton de Carvalho Lemos. Departamento de Farmácia da Universidade Es tadual da Paraíba, PB. Av. Floriano Peixoto, 1045, São José - CEP 58.110-001, Campina Grande, PB. E-mail: josiverton@hotmail.com

\section{Resumo}

Os acidentes causados por animais peçonhentos ainda constituem problema de Saúde Pública no Brasil. Embora a produção e a distribuição dos soros no país encontrem-se estabilizadas, há problemas relacionados à notificação de acidentes deste tipo em várias regiões. Considerando esta realidade, foi realizado um estudo transversal entre janeiro e dezembro de 2005, utilizando documentação indireta sobre os acidentes ofídicos ocorridos em Campina Grande e 80 municípios adjacentes à região, com o objetivo de conhecer o perfil epidemiológico e clínico deste tipo de caso. Todos os pacientes atendidos tiveram diagnóstico médico de acidente por serpentes realizado pelo Centro de Atendimento Toxicológico de Campina Grande (Ceatox-CG). Os dados foram coletados através da ficha de notificação do Sinan (Sistema Nacional de Notificação de Agravo do Ministério da Saúde). Para análise dos dados, foi utilizada estatística descritiva e os dados foram tabulados no programa Microsoft Excel 2003. Dos 1.443 atendimentos no Centro, 737 foram causados por animais peçonhentos e, destes, 277 foram provocados por serpentes peçonhentas e não peçonhentas. Os acidentes predominaram no sexo masculino, na faixa etária de 10 a 29 anos, principalmente em agricultores na zona rural, entre maio e novembro de 2005. O gênero Bothrops foi responsável pelo maior número de casos $(71,5 \%)$, e as extremidades superior e inferior do corpo foram os locais de maior predominância de ataques. Na extremidade inferior, foi atingido principalmente o pé. Em relação à gravidade, foram mais frequentes os acidentes classificados como leves causados pelo gênero Bothrops e apenas um paciente evoluiu para óbito. A média do tempo de atendimento, em horas, foi superior a 12 horas nos acidentes considerados graves, causados pelos gêneros Bothrops e Crotalus. Nossos resultados concordam com o perfil epidemiológico nacional dos acidentes ofídicos, atingindo com maior frequência o sexo masculino - trabalhadores rurais, na faixa etária produtiva de 10 a 49 anos - atingindo, sobretudo, os membros inferiores, $\mathrm{e}$ a maioria desses acidentes foi atribuída ao gênero Bothrops.

Palavras-chave: Picada de Cobra. Epidemiologia. Paraíba. Brasil. Cobras (BVS: Scielo). 


\section{Abstract}

Accidents caused by venomous animals are still a public health problem in Brazil. Although the production and distribution of antivenom is controlled, several regions have difficulties in reporting this type of accident. Considering such fact, a crosssectional study was carried out using indirect files on venom accidents that occurred in Campina Grande, along with 80 small towns comprising the region, from January to December 2005. The main objective was to outline a better epidemiological and clinical profile of these cases. All patients admitted to the Poisoning Information Center of Campina Grande (CEATOX-CG) had their injuries medically diagnosed as injuries caused by venomous snakes. Data collection was based on the use of a reporting form by the National Reporting System - SINAN (Sistema Nacional de Notificação de Agravo do Ministério da Saúde). Data analysis consisted of descriptive statistics using Microsoft Excel 2003. A number of 737 out of 1,443 patients who received care at the medical center had their cases related to venomous animals, and 277 of the accidents had been caused by venomous and non- venomous snakes. Most accidents involved men, from 10 to 29 years of age, including peasants in rural areas, from May to November 2005. Most of the cases (71.5\%) involved the Bothrops genus and upper and lower limbs, especially feet. As to severity, most of the cases were classified as mild, caused by the Bothrops genus, and only one patient died. The average length of time spent in providing care to the victims was over 12 hours in severe cases caused by Bothrops and Crotalus. Our results agree with the national epidemiological profile of snakebites, which are more frequent amongst male peasants aged from 10 to 49 , affecting especially lower limbs, caused most often by the Bothrops genus.

Keywords: Snake Bite. Epidemiology. Paraíba. Brazil. Snakes. (BVS: Scielo).

\section{Introdução}

O estudo do ofidismo no Brasil teve início com os trabalhos desenvolvidos nos primórdios do século XX por Vital Brazil no Instituto Serumterápico, hoje Instituto Butantan. Ao iniciar a produção de soros, este pesquisador introduziu os "Boletins para Observação dos Acidentes Ofídicos", porém até a década de 80 os estudos de notificação eram localizados, sendo realizado principalmente na região Sudeste. Por outro lado, havia deficiência na produção do soro. Em maio de 1986, várias medidas foram instituídas pelo Ministério da Saúde, dentre as quais podemos citar a criação do Programa Nacional de Ofidismo, na antiga Secretária de Ações Básicas da Saúde (SNABS/MS). Os acidentes ofídicos passaram então a ser de notificação obrigatória no país, permitindo uma relação de troca de informações epidemiológicas por soro entre as Secretarias Estaduais e o Ministério da Saúde. Com a implantação deste sistema aprimoraram-se os dados sobre ofidismo, mostrando características epidemiológicas e clínicas que permitiram o planejamento de ações de controle.

O Brasil apresenta diversas famílias de serpentes. Dentre estas, somente duas abrangem as serpentes consideradas peçonhentas: a família Viperidae, destacando-se a subfamília Crotalinae, à qual pertencem os gêneros Crotalus (Cascavel), Bothrops (Jararaca) e Lachesis (Surucucu); e a família Elapidae, que engloba o gênero Micrurus, cujas espécies são conhecidas popularmente por corais verdadeiras ${ }^{1}$.

É de grande relevância que se conheça o gênero a que pertence a serpente para que se possa tomar medidas terapêuticas fundamentais em caso de acidente. Contudo, o diagnóstico é feito, em geral, através dos sinais e sintomas apresentados pelo paciente, em consequência das atividades tóxicas causadas pela inoculação da peçonha ${ }^{2}$.

Dentre os animais peçonhentos, as peçonhas das serpentes são, provavelmente, as mais complexas, pois contêm vinte ou mais componentes diferentes, sendo mais 
de $90 \%$ de seu peso seco constituído por enzimas, toxinas não enzimáticas, proteínas e proteínas não tóxicas. As frações não protéicas são representadas por carboidratos, lipídios, aminas biogênicas, nucleotídeos e aminoácidos livres ${ }^{3}$.

O conhecimento da composição dos venenos e seus principais efeitos sobre o organismo humano permitem ao médico reconhecer o gênero da serpente responsável pelo acidente e selecionar a soroterapia adequada, mesmo na ausência da serpente ${ }^{2}$.

A letalidade dos acidentados varia em diferentes regiões do mundo. Na Europa, Estados Unidos e Canadá, os acidentes ofídicos são relativamente raros. Cerca de 90\% dos 8.000 envenenamentos ocorridos por ano são hospitalizados, resultando entre 15 a 30 casos fatais. Na África, a frequência dos acidentes ofídicos é precariamente documentada. Dos 500.000 casos de acidentes ofídicos, $40 \%$ são hospitalizados, resultando em 20.000 óbitos por ano. Na Ásia, principalmente no Paquistão, na Índia e na Birmânia, os acidentes ofídicos provocam de 25.000 a 35.000 óbitos por ano, sendo causados por uma das serpentes mais importantes do mundo: a Vipera russelli. No Japão a incidência geral é de aproximadamente $1 / 100.000$ habitantes e a letalidade é inferior a $1 \% 4$.

Entre os países sul-americanos, o Brasil é o que apresenta maior número de acidentes/ano ${ }^{5}$. O território brasileiro conta com 250 espécies de serpentes, sendo 70 delas consideradas peçonhentas ${ }^{6}$. Somente no ano de 2005 foram notificados pelo Sistema de Informação de Agravos de Notificação (Sinan) 97.244 envenenamentos por animais peçonhentos, dentre os quais as serpentes contribuíram com 28.702 casos $(29,52 \%)^{7}$.

No Brasil os dados sobre acidentes por animais peçonhentos são coletados através de sistemas de notificação como: Sistema de Informação de Agravos de Notificação (Sinan/MS), Sistema de Nacional de Informações Tóxico-Farmacológicas (Sinitox/ Fiocruz/MS), Sistema de Informações Hospitalares do Sistema Único de Saúde/MS e o
SIM (Sistema de Informações sobre Mortalidade/MS). Apesar de todos estes sistemas, os dados epidemiológicos disponíveis não retratam a real magnitude do problema, provavelmente devido à subnotificação dos casos, tendo em vista, entre outros fatores, as dificuldades de acesso aos serviços de saúde de muitos municípios brasileiros.

O presente estudo pretende contribuir para a caracterização do perfil epidemiológico e clínico dos acidentes ofídicos no município de Campina Grande e cidades circunvizinhas.

\section{Metodologia}

\section{Local do estudo}

O município de Campina Grande está localizado no Agreste Paraibano, distante 120 km de João Pessoa, capital do Estado da Paraíba. Segundo dados do Instituto Brasileiro de Geografia Estatística - IBGE, este possui uma área territorial de $671 \mathrm{Km}^{2}$ e uma população constituída de 379.871 habitantes, sendo que $46,87 \%$ são homens e 53,2\% são mulheres ${ }^{8}$. Por dispor de uma posição geográfica privilegiada, Campina Grande é um polo de convergência, com aproximadamente 232 municípios, tanto da Paraíba, quanto de estados vizinhos, cujos habitantes se deslocam para o município em busca dos serviços oferecidos, entre os quais os de saúde.

Esta pesquisa foi realizada no Centro de Assistência e Informação Toxicológica (Ceatox), serviço oferecido pelo Departamento de Farmácia da Universidade Estadual da Paraíba - UEPB em parceria com o Hospital Regional de Urgência e Emergência de Campina Grande (HRUECG). Este serviço, que é considerado de referência para o Município de Campina Grande e o Estado da Paraíba, é dirigido à população em geral, funcionando em regime de plantão permanente.

\section{Desenho e população de estudo}

Trata-se de um estudo transversal, utilizando documentação indireta, sobre 
os acidentes ofídicos ocorridos em Campina Grande e cidades circunvizinhas, entre janeiro e dezembro de 2005. Todos os pacientes incluídos no estudo apresentavam diagnóstico médico de acidentes por serpentes no Ceatox-CG. Os dados foram coletados através das fichas de notificação do Sinan para Animais Peçonhentos, do Ministério da Saúde.

\section{Variáveis de estudo}

Foram analisadas as seguintes variáveis: demográficas, socioeconômicas, e as relativas ao acidente, ao gênero da serpente; $\mathrm{e}$ as clínicas.

A gravidade do envenenamento foi classificada conforme recomendação do Ministério da Saúde: leve, moderado ou grave, nos acidentes botrópicos e crotálicos, e grave no acidente elapídico ${ }^{9}$.

\section{Análise dos dados}

Os dados foram tabulados e analisados através de estatística descritiva no Microsoft Excel, versão 2003.

\section{Resultados}

No período estudado foram atendidos e notificados 1.443 casos de intoxicações por biocidas, medicamentos, animais peçonhentos, plantas tóxicas, drogas de abuso e produtos químicos. Destes, 737 casos foram causados por animais peçonhentos, sendo que 277 foram causados por serpentes (Tabela 1).

Na Tabela 2 observa-se a caracterização dos acidentes ofídicos atendidos e notificados no Ceatox-CG, segundo as variáveis sociodemográficas. Os acidentes foram mais frequentes no sexo masculino, com 199 casos $(71,8 \%)$. Através da análise de Correlação de Pearson, avaliamos a relação existente entre os grupos, que apresentou correlação positiva $(r=0,883)$. Com relação à faixa etária, observou-se maior frequência de acidentes entre 10 e 19 anos, para ambos os sexos, contribuindo para $22,8 \%$ das ocorrências. Observa-se, também, que para a variável grau de instrução, prevaleceu o número de casos ignorados: 154 (55,6\%).

No tocante à ocupação, constatou-se que a maioria dos acidentes ocorreu em agricultores, 127 casos (45,8\%), e na zona rural, 205 casos (74\%).

Os acidentes ofídicos ocorreram durante todos os meses, ainda que de forma irregular. Observou-se um pequeno aumento nas notificações no período de maio a novembro, e um declínio de casos nos meses de dezembro a abril. Contudo, a maior incidência de acidentes ofídicos

Tabela 1 - Prevalência dos casos de intoxicações e acidentes com plantas e animais peçonhentos no Ceatox-CG durante o ano de 2005.

Table 1 - Prevalence of cases of poisoning and accidents with venomous plants and animals in Ceatox-CG during 2005.

\begin{tabular}{lccccc}
\hline Agente Tóxico & \multicolumn{3}{l}{ Casos de intoxicação por trimestre no ano de 2005 (N=277) } & \multirow{2}{*}{ Total (\%) } \\
\cline { 2 - 5 } & $1^{\circ}$ & $2^{\circ}$ & $3^{\circ}$ & $4^{\circ}$ & \\
\hline Animais Peçonhentos e não Peçonhentos & $\mathrm{n}$ & $\mathrm{n}$ & $\mathrm{n}$ & $\mathrm{n}$ & \\
Medicamentos & 112 & 185 & 242 & 188 & $727(50,5)$ \\
Alimentos & 34 & 45 & 68 & 56 & $203(14,0)$ \\
Drogas de Abuso & 50 & 55 & 43 & 14 & $162(11,2)$ \\
Agrotóxicos & 23 & 45 & 38 & 15 & $121(8,4)$ \\
Produtos Químicos & 11 & 20 & 34 & 28 & $93(6,4)$ \\
Agente Ignorado & 14 & 20 & 25 & 18 & $77(5,3)$ \\
Plantas Tóxicas & 9 & 14 & 12 & 5 & $40(2,8)$ \\
Produto de Uso Veterinário & 4 & 2 & 5 & 2 & $13(0,9)$ \\
\hline Total & 3 & 2 & 1 & 1 & $7(0,5)$ \\
\hline
\end{tabular}


Tabela 2 - Caracterização dos casos de acidentes ofídicos atendidos e notificados no Ceatox-CG, segundo as variáveis socioeconômicas e demográficas. Campina Grande, Paraíba, 2005.

Table 2 - Description of cases of snakebites that received care and were reported by Ceatox-CG, according to socio-economic and demographic variables. Campina Grande, Paraíba, 2005.

\begin{tabular}{|c|c|c|c|}
\hline \multicolumn{4}{|c|}{ Distribuição dos casos de acidentes ofídicos por sexo no ano de 2005 ( $n=277)$} \\
\hline $\begin{array}{l}\text { Variáveis demográficas } \\
\text { e socioeconômicas }\end{array}$ & $\begin{array}{c}\text { Homem } \\
\mathrm{n}(\%)\end{array}$ & $\begin{array}{l}\text { Mulher } \\
\mathrm{n}(\%)\end{array}$ & $\begin{array}{l}\text { Prevalência } \\
\text { (\%) }\end{array}$ \\
\hline \multicolumn{4}{|l|}{ Faixa Etária (anos) } \\
\hline $1-9$ & $10(3,6)$ & $8(2,9)$ & 6,5 \\
\hline $10-19$ & $45(16,3)$ & $18(6,5)$ & 22,7 \\
\hline $20-29$ & $36(13,0)$ & $11(4,0)$ & 17,0 \\
\hline $30-39$ & $34(12,3)$ & $12(4,3)$ & 16,6 \\
\hline $40-49$ & $22(7,9)$ & $11(4,0)$ & 12,0 \\
\hline 50 e $>$ & $51(18,4)$ & $18(6,5)$ & 24,9 \\
\hline Ignorada & $1(0,3)$ & - & 0,36 \\
\hline \multicolumn{4}{|l|}{ Grau de instrução } \\
\hline Analfabeto & $20(7,2)$ & $8(2,9)$ & 10,0 \\
\hline 1o Grau incompleto & $6(2,2)$ & $3(1,09)$ & 3,2 \\
\hline 1o Grau completo & $44(15,9)$ & $20(7,2)$ & 23,1 \\
\hline 2o Grau completo & $8(2,9)$ & $3(1,09)$ & 4,0 \\
\hline 2o Grau incompleto & $4(1,45)$ & $4(1,45)$ & 3,0 \\
\hline Superior & - & - & - \\
\hline Ignorado & $119(43,0)$ & $35(12,6)$ & 55,6 \\
\hline Não se aplica & $1(0,3)$ & $2(0,7)$ & 1,0 \\
\hline \multicolumn{4}{|l|}{ Ocupação } \\
\hline Estudante & $31(11,2)$ & $14(5,1)$ & 16,3 \\
\hline Agricultor & $102(36,8)$ & $25(9,0)$ & 45,8 \\
\hline Menor & $2(0,7)$ & $2(0,7)$ & 1,4 \\
\hline Aposentado & $3(1,1)$ & $3(1,1)$ & 2,2 \\
\hline Outros & $7(2,5)$ & $16(5,8)$ & 8,3 \\
\hline Ignorado & $49(17,7)$ & $23(8,3)$ & 26,0 \\
\hline \multicolumn{4}{|l|}{ Zona de ocorrência } \\
\hline Rural & $144(52,0)$ & $61(22,0)$ & 74,0 \\
\hline Urbana & $45(16,2)$ & $21(7,6)$ & 23,8 \\
\hline Ignorada & $5(1,8)$ & $1(0,4)$ & 2,2 \\
\hline
\end{tabular}

NOTA: Os valores absolutos e percentuais de cada variável da tabela totalizam, respectivamente, 277 e $100 \%$. NOTE: The absolute and percent values of each variable in the table total respectively, 277 and 100\%

ocorreu no mês de agosto, com 37 casos $(13,4 \%)$, seguidos dos meses de julho, com $34(12,3 \%)$, e de setembro e novembro, 33 $(12,0 \%)$ (Figura 1).

Os municípios com maior ocorrência de acidentes foram: Campina Grande, representando $10,8 \%$, Taperoá $(6,8 \%)$, Soledade
$(6,4 \%)$, Boa Vista $(4,3 \%)$ e Barra de Santana $(4,1 \%)$; os demais municípios apresentaram índice menor que $4 \%$ (Figura 2).

Com relação à região anatômica, na qual ocorrem as picadas das serpentes, as extremidades foram os locais de maior predominância, com 246 casos (88,8\%), sendo 


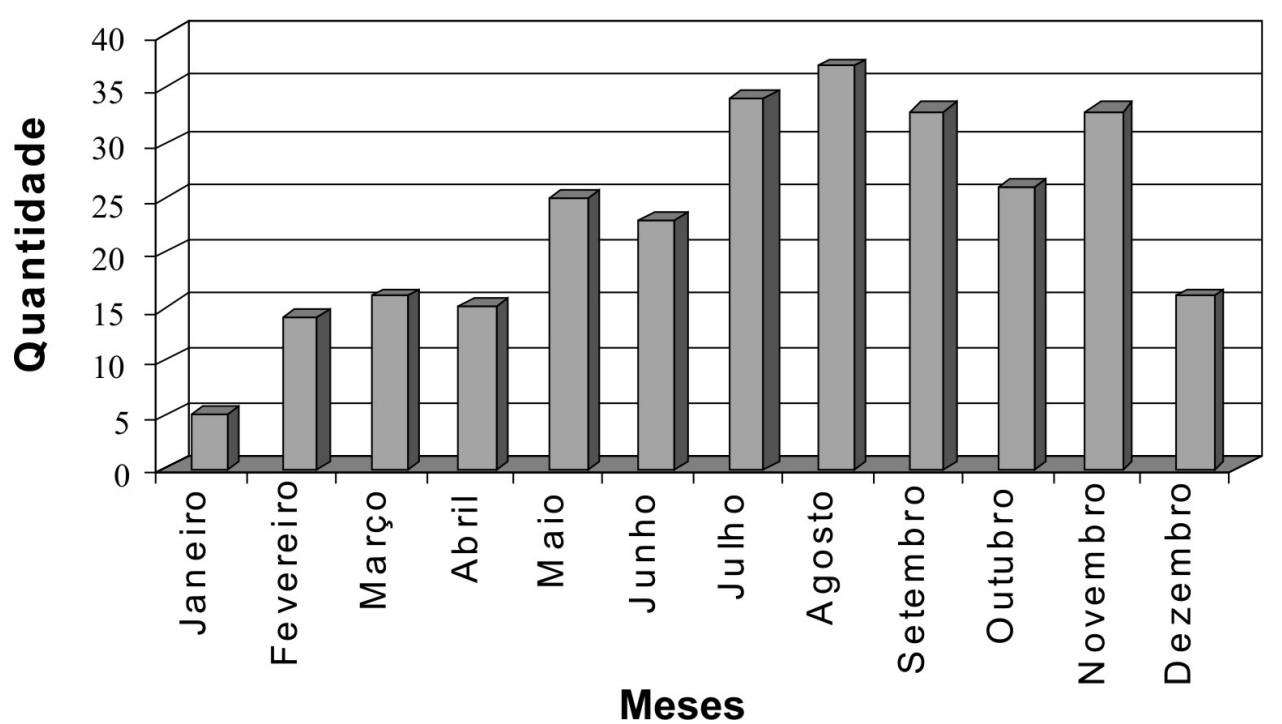

Figura 1 - Distribuição dos casos de acordo com a sazonalidade dos acidentes ofídicos atendidos no Ceatox-CG, no período de janeiro a dezembro de 2005.

Figure 1 - Case distribution of snakebites that received care at Ceatox-CG per seasonality, in the period from January to December 2005.

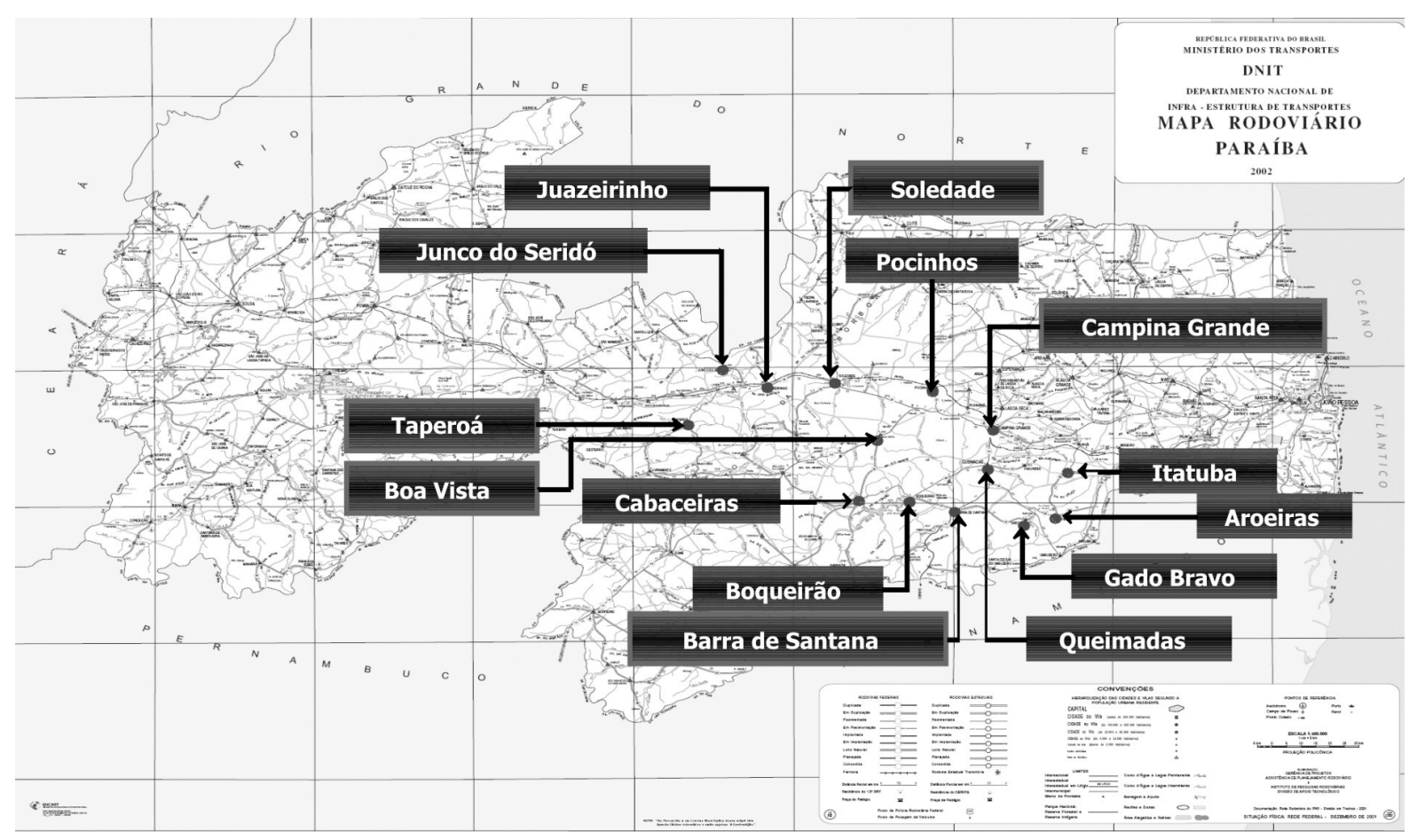

Figura 2 - Distribuição dos acidentes ofídicos por municípios, no período de janeiro a dezembro de 2005.

Figure 2 - Distribution of snakebites per municipality, in the period from January to December 2005.

65 deles $(26,4 \%)$ na extremidade superior e $181(73,6 \%)$ na extremidade inferior. $\mathrm{Na}$ extremidade superior, o dedo da mão foi o local mais acometido, 37 ocorrências (56,9\%); na extremidade inferior foi o pé, com 134 casos $(74,0 \%)$ (Tabela 3$)$. 
Tabela 3 - Distribuição de frequência dos acidentes ofídicos atendidos no Ceatox-CG de acordo com a região anatômica da picada, entre janeiro e dezembro de 2005.

Table 3 - Frequency distribution of snakebites that received care at Ceatox-CG according to the anatomical region of the bite, between January and December 2005.

\begin{tabular}{lcc}
\hline Local da Picada & \multicolumn{2}{c}{ Número de ocorrências } \\
\cline { 2 - 3 } & Freq. Absoluta $(\mathrm{n})$ & Freq. Relativa $(\%)$ \\
\hline Cabeça & 2 & 0,72 \\
Braço & 0 & 0,00 \\
Antebraço & 4 & 1,44 \\
Mão & 28 & 10,11 \\
Dedo da mão & 37 & 13,36 \\
Tronco & 1 & 0,36 \\
Coxa & 0 & 0,00 \\
Perna & 17 & 6,14 \\
Pé & 134 & 48,38 \\
Dedo do pé & 30 & 10,83 \\
Ignorado & 24 & 8,66 \\
\hline TOTAL & 277 & 100 \\
\hline
\end{tabular}

Tabela 4 - Distribuição dos acidentes ofídicos de acordo com: o tipo de serpentes, o tempo médio entre o acidente e o atendimento, a classificação dos casos e a evolução, no Ceatox-CG, entre janeiro e dezembro de 2005.

Table 4 - Distribution of snakebites according to the type of snake, average time span between accident and care, classification of cases, and outcome at Ceatox-CG, between January and December 2005.

\begin{tabular}{lccccc}
\hline Tipo de Acidente & \multicolumn{2}{c}{ Classificação dos casos $(\mathrm{N}=277)$} & \multicolumn{2}{c}{ Evolução } \\
& Leve & Moderado & Grave & Cura & Óbito \\
\hline Botrópico & 140 & 46 & 12 & 197 & 1 \\
Crotálico & 0 & 2 & 5 & 7 & 0 \\
Elapídico & 0 & 0 & 3 & 3 & 0 \\
*SNP & 0 & 0 & 0 & 69 & 0 \\
\hline TOTAL & 140 & 48 & 20 & 276 & 1 \\
\hline
\end{tabular}

Com base no diagnóstico e/ou identificação das serpentes, os gêneros responsáveis pelos acidentes foram: Bothrops (198 casos - $71,3 \%$ ), serpentes não peçonhentas (69 casos - 24,9\%), Crotalus (7 casos - 2,5\%) e Micrurus (3 casos - 1,1\%) (Tabela 4).

Nos acidentes por serpentes do gênero Bothrops, os casos leves prevaleceram, com 140 registrados $(70,7 \%)$, seguidos de 46 moderados (23,3\%) e 12 graves $(6,0 \%)$; nos acidentes por serpentes do gênero Crotalus, ocorreram 2 casos moderados $(28,7 \%)$ e 5 graves $(71,4 \%)$. Os acidentes por serpentes Micrurus foram classificados como graves. A evolução para cura predominou, havendo apenas um óbito, causado por serpente do gênero Bothrops, com um coeficiente de letalidade de $0,5 \%$. Observa-se, ainda, na Tabela 4, que $24,9 \%$ dos casos atendidos foram causados por serpentes não peçonhentas ou por serpentes peçonhentas que não causaram envenenamento (picada seca), ou seja, os pacientes não apresentaram qualquer anormalidade. Quanto às 
espécies das serpentes consideradas não peçonhentas, predominaram acidentes com corre-campo (Philodryas nattereri) e cobra verde (Philodryas olfersii), ambas pertencentes à família Colubridae.

A média geral do tempo, em horas, decorrido entre o acidente e o atendimento, baseado na gravidade foi de $4,3( \pm 2,4), 5,3$ $( \pm 3,7)$ e $26,5( \pm 29,9)$ nos casos leves, moderados e graves, respectivamente.

\section{Discussão}

No município de Campina Grande, o Centro de Assistência e Informação Toxicológica constitui a unidade de referência para o atendimento de acidentes ofídicos. Sua relevância fica demonstrada pelo número de casos atendidos nesta unidade.

O predomínio de acidentes entre o sexo masculino, na faixa etária entre 10 a 19 anos, pode ser devido à precoce inserção no trabalho agrícola das pessoas nesta faixa etária, muito provavelmente com vistas a contribuir para o aumento da renda fami$\operatorname{liar}^{10}$. No estado do Ceará, Feitosa et al. ${ }^{11}$, no Amazonas, Borges et al. ${ }^{12} \mathrm{e}$, na região do Noroeste de São Paulo, Rojas et al. ${ }^{13}$ encontraram dados semelhantes. Fonte do Ministério da Saúde de $2005^{14}$ mostra que, quanto ao grau de instrução, predominaram os casos ignorados (213), o que se assemelha aos nossos resultados.

Nesta pesquisa observou-se a prevalência da atividade agropecuária e da zona rural. Na região Nordeste, principalmente no interior, na época de plantio e colheita, observa-se uma maior movimentação de trabalhadores rurais. Assim, provavelmente pode haver uma relação direta entre o aumento de acidentes por serpentes e a época destinada ao plantio e à colheita da safra agrícola. Estas informações reforçam a conotação do acidente ofídico como acidente de trabalho, uma vez que o seu incremento coincide com o deslocamento do trabalhador rural para as atividades do campo $^{11,15}$

Os resultados confirmam a observação feita por Theakston e Griffiths ${ }^{16}$, que relatam que no mundo inteiro a atividade agrícola se apresenta como um fator de risco para a ocorrência de acidentes ofídicos.

Albuquerque $^{15}$ e Swaroop e Grab ${ }^{17}$ justificam o fato afirmando que os índices de acidentes por serpentes obedecem a uma variação regional considerável, ou seja, estudos regionalizados podem demonstrar diferenças marcantes como as que ocorrem entre a região Sul/Sudeste e a região Nordeste. No Sul/Sudeste os acidentes predominam nos meses de outubro a abril, caracterizados por um período chuvoso e quente, com um recesso no inverno, enquanto na região Nordeste há um aumento nos meses de maio a setembro, seguido de decréscimo a partir de outubro.

Em Goiás, Pinho et al. ${ }^{18}$ relataram que entre os meses de abril e outubro, os acidentes foram mais frequentes (entre $1998 \mathrm{e}$ 2000), coincidindo com o período de maior pluviosidade e temperatura, bem como de maior atividade agropecuária nesta região.

As vítimas foram picadas mais frequentemente nos membros inferiores, com maior frequência nos pés. Em São Paulo, Ribeiro et al. ${ }^{19}$, e no Acre, Moreno et. al. ${ }^{20}$ relataram, também, que a região anatômica mais frequentemente atingida foi a do pé $(43,1 \%)$, seguindo-se a perna $(25,7 \%)$ e a mão (16,5\%).

Portanto, o uso de equipamentos de proteção específicos, como perneiras, botas de cano alto, luvas ou instrumentos para retirar entulhos e remover o mato, como enxadas e pás, poderiam evitar cerca de 50 a $75 \%$ dos $\operatorname{casos}^{21,22}$.

Nesta pesquisa verificou-se que o gênero Bothrops foi responsável pela maioria dos acidentes envolvendo serpentes peçonhentas. Devido à capacidade de se adaptar a diferentes tipos de ambientes ${ }^{23}$, as serpentes desse gênero podem ser encontradas nos mais diversos ecossistemas.

Dentre os estudos epidemiológicos realizados em alguns estados do Brasil, podemos citar o de Moreno et al..$^{20}$, realizado no Acre no ano de 2002. Neste, o gênero Bothrops foi responsável pelo maior número de acidentes, com 109 casos (75,7\%), seguido pelo 
gênero Lachesis com 3 (2,1\%) e o gênero Micrurus com $1(0,7 \%)$. Bochner e Struchiner ${ }^{10}$, num trabalho de revisão da literatura sobre a epidemiologia dos acidentes ofídicos no Brasil nos últimos cem anos, concluíram que a maioria desses acidentes é causada por serpentes do gênero Bothrops.

Os casos classificados como grave foram atendidos, em média, mais tardiamente do que os demais, o que sugere que o tratamento tardio seja fator de mau prognóstico, o que é plenamente explicável, pois o soro deve ser administrado o mais precocemente possível, com o intuito de neutralizar as atividades do veneno ${ }^{15}$.

De acordo com o Ministério da Saúde ${ }^{9}$, uma vez indicada a quantidade de soro a ser administrada, esta deve ser aplicada em dose única.

Além disso, na região Nordeste há pacientes que demoram várias horas para procurar atendimento na unidade de saúde do seu município, como também podem não receber o tratamento adequado e acabam sendo transferidos para unidades mais complexas de outra cidade. O extenso lapso temporal entre $\mathrm{o}$ acidente $\mathrm{e} \mathrm{o}$ atendimento pode determinar a evolução para um quadro mais grave.

No Ceará, diferentemente dos nossos resultados, Feitosa et al. ${ }^{11}$, avaliando aspectos epidemiológicos dos acidentes ofídicos entre 1992 e 1995, relatam que os óbitos foram mais frequentes nos acidentes por serpentes do gênero Crotalus. Além disso, nos anos de 2005 e 2006, dados do Ministério da Saúde notificaram 75 e 50 óbitos por serpentes do gênero Bothrops, e para o gênero Crotalus 20 e 12 óbitos, respectivamente. Contudo, o coeficiente de letalidade é maior para os acidentes Crotálicos do que para os acidentes Botrópicos ${ }^{7,14}$.

\section{Conclusão}

Conclui-se que os acidentes envolvendo animais peçonhentos, notificados no Ceatox-CG, apresentaram elevada prevalência em relação a outros agentes. A epidemiologia dos acidentes ofídicos corrobora para um perfil no Brasil que evidencia maior frequência em trabalhadores rurais do sexo masculino na faixa etária produtiva de $10 \mathrm{a}$ 49 anos; atingem, sobretudo, os membros inferiores; e a maioria desses acidentes é atribuída ao gênero Bothrops. Vale ressaltar a importância do correto preenchimento da ficha de notificação e a necessidade de um tratamento precoce para as vítimas de acidentes ofídicos, utilizando, sempre que possível, a via endovenosa para administração do soro específico com o menor tempo possível entre o acidente e o atendimento. A inclusão de acidente ofídico na lista de doenças ocupacionais e a sua adequada vigilância poderiam representar um avanço em Saúde Pública, não somente por determinar menor incidência, mas também porque o precoce e correto encaminhamento dos que se acidentam pode diminuir a limitação temporária e às vezes definitiva na capacidade de trabalho, eventualmente causada por essa condição, assim como sua mortalidade e letalidade.

\section{Referências}

1. Melgarejo, AR. Serpentes Peçonhentas do Brasil. In: Cardoso, LC et al. Animais Peçonhentos no Brasil: biologia, clínica e terapêutica dos acidentes. São Paulo: Savier; 2003, p. 33-61.

2. Azevedo-Marques M, Cupo P, Hering SE. Acidentes por animais peçonhentos: Serpentes peçonhentas. Medicina 2003; 36: 480-9.
3. França, FO DE S, Málaque, CMS. Acidente botrópico. In: Cardoso, LC et al. Animais Peçonhentos no Brasil: biologia, clínica e terapêutica dos acidentes. São Paulo: Savier; 2003, p. 72-86.

4. Chippaux JP. Snakebites: appraisal of the global situation. Bull World Health Organ 1998; 76(5): 515-24. 
5. World Health Organization. Progress in the characterization of venoms and standardization of antivenoms. Geneva; 1981. (WHO offset Publication, 58).

6. Pinho, FMO, Pereira, I. D. Ofidismo. Rev Assoc Med Bras 2001; 47(1): 24-9.

7. SINAN-Sistema Nacional de Agravos de Notificação. Estatística 2006. Ministério da Saúde. Disponível em http://dtr2004.saude.gov.br/sinanweb. [Acessado em 20 de março de 2008].

8. IBGE-Instituto Brasileiro de Geografia e Estatística. Ministério do Planejamento Orçamento e Gestão [Online]. Brasília, Brasil; 2007. Disponível em http//www. ibge.gov.br/ cidadesat/ default.php. [Acessado em 20 de março de 2008].

9. BRASIL. Ministério da Saúde. Manual de diagnóstico e tratamento de acidentes por animais peçonhentos. $2^{\circ}$ edição; [on-line]. Brasília: Fundação Nacional de Saúde; 2001. Disponível em http://www.funasa.gov.br/pub/ pub00.htm. [Acessado em 10 de junho de 2007].

10. Bochner R, Struchiner CJ. Aspectos ambientais e sócioeconômicos relacionados à incidência de acidentes ofídicos no Estado do Rio de Janeiro de 1990 a 1996: uma análise exploratória. Cad Saúde Pública 2004; 20(4): 976-85.

11. Feitosa RFG, Melo IMLA, Monteiro HSA. Epidemiologia dos acidentes por serpentes peçonhentas do Estado do Ceará - Brasil. Rev Soc Bras Med Trop 1997; 30(4): 295301.

12. Borges, CC, Sadahiro M, Santos Ma C DOS. Aspectos epidemiológicos e clínicos dos acidentes ofídicos ocorridos nos municípios do Estado do Amazonas. Rev Soc Bras Med Trop 1999; 32(6): 637-46.

13. Rojas, C A, Gonçalves, MR, Almeida, Santos, SM. Epidemiologia dos acidentes ofídicos na região noroeste do estado de São Paulo, Brasil. Rev Bras Saúde Prod An 2007; 8(3): 193-204.
14. SINAN - Sistema Nacional de Agravos de Notificação. Estatística 2005. Ministério da Saúde. Disponível em http://dtr2004.saude.gov.br/sinanweb. [Acessado em 20 de março de 2008].

15. Albuquerque HN. Perfil clínico e epidemiológico dos acidentes ofídicos notificados no estado da Paraíba [dissertação de mestrado]. Campina Grande: Universidade Estadual da Paraíba; 2002.

16. Theakston, RDG, Warrell, DAB, Griffiths E. Report of a WHO workshop on the standardization and control of antivenoms. Tox 2003; 41: 541-57.

17. Swarrop S, Grad B. Snakebite mortality in the world. Bull World Health Organ 1954; 10: 35-76.

18. Pinho FMO, Oliveira ES, Faleiros F. Acidente ofídico no estado de Goiás. Rev Assoc Med Bras 2004; 50(1): 93-9.

19. Ribeiro, LA, Jorge, MT, Iversson, LB. Epidemiologia do acidente por serpentes peçonhentas: estudo de casos atendidos em 1988. Rev Saúde Publica 1995; 29(5): 3808 .

20. Moreno E, Queiroz-Andrade M, Silva, RM, Neto JT. Características clínico-epidemiologicas dos acidentes ofídicos em Rio Branco, Acre. Rev Soc Bras Med Trop 2005; 38(1): 15-21.

21. Brazil V. A defesa contra o ophidismo. São Paulo: Editora Pocai \& Weiss, 1911.

22. Campbell JA, LamarWW. The venomous reptiles of Latin America. New York: Cornell University Press; 1989.

23. Barravieira B. Venenos: aspectos clínicos e terapêuticos dos acidentes por animais peçonhentos. Rio de Janeiro: EPUD; 1999.

24. Fan, HW. Soroterapia. In. Cardoso, Luiz da Costa et al. Animais Peçonhentos do Brasil: biologia, clínica e terapêutica dos acidentes. São Paulo: Editora Sarvier; 2003. p. 381-93.

Recebido em: 26/10/07

Versão final reapresentada em: 21/11/08 Aprovado em: 05/01/09 